\title{
The Case for Resource Sharing in Scientific Workflow Executions
}

\author{
Ricardo Oda $^{1}$, Daniel Cordeiro ${ }^{1}$, Rafael Ferreira da Silva ${ }^{2}$ \\ Ewa Deelman ${ }^{2}$, Kelly R. Braghetto ${ }^{1}$
}

\author{
${ }^{1}$ Instituto de Matemática e Estatística - Universidade de São Paulo (USP) \\ São Paulo, SP - Brazil \\ ${ }^{2}$ Information Sciences Institute - University of Southern California (USC) \\ Marina del Rey, CA - USA \\ \{odaric, danielc,kellyrb\}@ime.usp.br, \{rafsilva, deelman\}@isi.edu
}

\begin{abstract}
Scientific workflows have become mainstream for conducting largescale scientific research. The execution of these applications can be very costly in terms of computational resources. Therefore, optimizing their resource utilization and efficiency is highly desirable, even in computational environments where the processing resources are plentiful, such as clouds. In this work, we study the case of exploring shared multiprocessors within a single virtual machine. Using a public cloud provider and real-world applications, we show that the use of dedicated processors can lead to sub-optimal performance of scientific workflows. This is a first step towards the creation of a self-aware resource management system inline with the state-of-the-art multitenant platforms.
\end{abstract}

\section{Introduction}

Modern scientific experiments are automatized by means of workflows systems. Workflow systems have received increased attention from the research community since their first appearance in the 1980s. Scientific workflows have become mainstream for conducting large-scale scientific research [Taylor et al. 2007]. Workflows can be seen as "process networks that are typically used as 'data analysis pipelines' or for comparing observed and predicted data, and that can include a wide range of components, e.g. for querying databases, for data transformation and data mining steps, for execution of simulation codes on high-performance computers, etc." [Ludäscher et al. 2006].

According to [Callaghan et al. 2011], scientific workflow applications may include both high-performance computing (HPC) and high-throughput computing (HTC) tasks. While HPC couples high-speed networks with high-performance computers to provide specialized collections of resources to applications, HTC is characterized by the use of many computing resources over long periods of time to accomplish a computational task. In the past decade, HPC and HTC techniques have been used to probe vast amounts of raw scientific data. The processing of such data is very costly in terms of computational resources. Therefore, it is highly desirable that scientific applications maximize resource utilization and thereby efficiency, even in computational environments where the processing resources are plentiful, such as in multiprocessor computers, grids, and clouds.

Scientific workflow management systems (SWfMSs) are a class of tools created to ease the development, management, and execution of complex workflows. SWfMSs 
allow scientists to execute their HPC and HTC applications (expressed as workflows) seamlessly on a range of distributed platforms. In order to support a large variety of scientific applications, SWfMSs must make assumptions about the tasks they will execute and the underlying execution environment. Notably, most SWfMSs assume that each task requires a fully dedicated processor for execution.

The one-task-per-processor assumption is mainly justified by software requirements related to the execution on clusters and grids. In these platforms, users often have exclusive access to the computational resources. However, with the large use of virtual machines (VMs) on multitenant platforms, where cloud computing is the most prominent example, similar assumptions may no longer be reasonable. A single physical machine can multiplex the execution of several VMs, without the user being aware of this.

In this work, we study the case of exploring shared multiprocessors within a single VM. Using a public cloud computing provider and well-studied scientific applications, we show that the use of dedicated processors can lead to sub-optimal execution performance of scientific workflows. This work is a first step towards the creation of a multipurpose, self-aware resource management system inline with the state-of-the-art multitenant platforms.

\section{Scientific Workflows}

Scientific workflows allow researchers to express multi-step computational tasks, for example: retrieve data from an instrument or a database, reformat the data, run analyses, and post-process results. A scientific workflow is basically composed of data processing tasks and the connections existing among these tasks. A workflow model is an abstract representation of a workflow that defines the tasks that must be performed and their (partial) execution order. A task is an atomic unit of work in the workflow model. The execution order of the tasks can be defined in terms of the dependency relationships that may exist among them, or in function of their data transfers (i.e., data flow). Therefore, a workflow model is often represented as a graph in a formal framework or in a workflow specification language. A workflow instance is a specific execution of a workflow, i.e. is an instantiation of a workflow model with its own input data and configuration parameters.

In this work, a scientific workflow is modeled as a Directed Acyclic Graph (DAG), where nodes represent individual computational tasks and the edges represent data and control dependencies between tasks. Figure 2 shows an example of workflows modeled as DAGs. Despite their simplicity, DAGs enable the representation of the most common data flow patterns used to model scientific workflows, such as pipeline, data distribution (data parallelism), and data aggregation (data synchronization) - all of them illustrated in the workflow model of Figure 2a.

\subsection{Execution on Cloud Computing Platforms}

Several works [Hoffa et al. 2008, Juve et al. 2009, Deelman et al. 2008] have shown that clouds are a viable computing platform for the execution of scientific applications. They can provide a large amount of on-demand, elastic resources at lower costs than those involved in acquiring and maintaining a high-performance cluster or supercomputer. Public cloud computing platforms offer virtualized resources that can be provisioned and released at any time. As a result, one can adapt the set of computational resources to the 
specific resource requirements of workflow instances, even when the requirements vary during the instances execution [Deelman et al. 2012].

Due to virtualization, scheduling techniques similar to the classic ones used to execute scientific workflows in grids can be extended to clouds. These techniques are designed to optimize specific performance goals such as total completion time (makespan) and average completion time of tasks. In addition, there is a clear tradeoff between performance and cost. Performance can be improved by provisioning additional resources at the expense of high costs. Finding a solution that satisfies both criteria (multi-objective problem) is known to be an NP-hard problem, thus several heuristics have been developed to tackle this problem [Malawski et al. 2012, Fard et al. 2012]. In this context, optimizing the use o computational resources (e.g., by using resource sharing) is a feasible approach to reduce costs with minimal impact on the overall workflow execution performance.

\subsection{Scientific Workflow Management System (SWfMS)}

A scientific workflow management system (SWfMS) is a software tool that supports the modeling, instantiation, execution, monitoring, and analysis of scientific workflows. Several SWfMSs [Wolstencroft et al. 2013, Ludäscher et al. 2006, Giardine et al. 2005, Deelman et al. 2015] have been developed to allow scientists to: (i) describe their applications as high-level abstractions decoupled from the specifics of workflow execution; and (ii) execute these applications seamlessly on possibly complex, multi-site distributed platforms that can accommodate large-scale executions.

Taverna [Wolstencroft et al. 2013], Kepler [Ludäscher et al. 2006], Galaxy [Giardine et al. 2005], and Pegasus [Deelman et al. 2015] are examples of open-source, general-purpose SWfMSs that have been largely used by the scientific community. Although these systems implement different automation approaches, most of them share common architectural components: $(i)$ Execution environment-represents the target execution infrastructure; (ii) Task Manager-abstracts the complexity of the physical platform and provides mechanisms to execute workflow tasks in a scalable, efficient, and robust manner; (iii) Workflow Engine-manages and monitors the state of the workflow execution; and (iv) Workflow Planner-maps high-level workflow descriptions onto distributed resources. In this work, we have particular interest in the resource manager (execution environment), which controls the software and hardware components used in the workflow execution.

\subsubsection{The Pegasus SWfMS}

We used the Pegasus SWfMS [Deelman et al. 2015] as a case study and a driver for this work. Pegasus bridges the scientific domain and the execution environment by automatically mapping high-level abstract workflows descriptions onto distributed resources. It manages data on behalf of the user, infers the required data transfers, registers data into catalogs, and captures performance information while maintaining a common user interface for workflow submission. In Pegasus, workflows are described abstractly as DAGs, where nodes represent individual computational tasks and the edges represent data and control dependencies between tasks, without any information regarding physical resources or physical locations of data and executables. The abstract workflow description 
is represented as a DAX (DAG in XML), describing all tasks, their dependencies, their required inputs, their expected outputs, and their invocation arguments.

Figure 1 shows an overview of the Pegasus architecture. The Workflow Mapper component of Pegasus is in charge of planning the workflow execution. During execution, Pegasus translates the abstract workflow into an executable workflow, determining the executables, data, and computational resources required for the execution. Pegasus maps executables to their installation paths or to a repository of stageable binaries defined in a Transformation Cata$\log$. Workflow execution with Pegasus includes data management, monitoring, and failure handling, and is managed by DAGMan [Frey 2002]. Individual workflow tasks are managed by a task scheduler

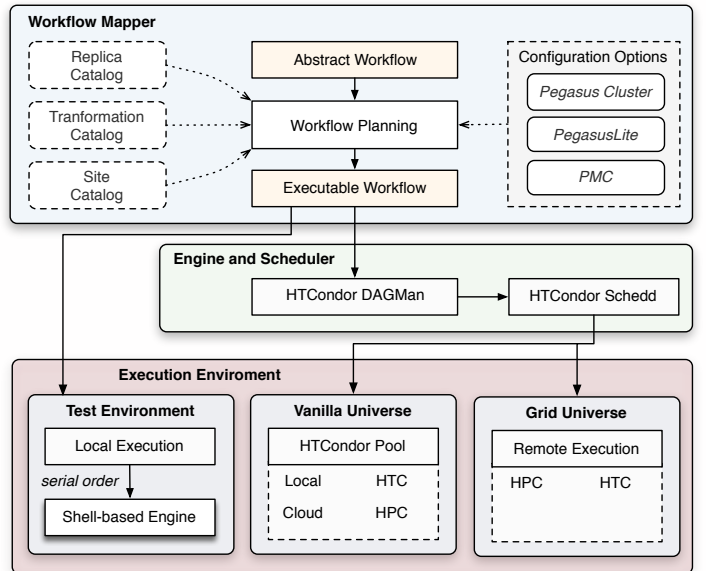

Figure 1. Overview of the Pegasus SWfMS architecture.

(HTCondor [Thain et al. 2005]), which supervises their execution on local and remote resources. HTCondor supports a number of different execution layouts. The mapping of abstract workflow tasks to executable workflow jobs depends on the underlying execution infrastructure. In this work, we target execution platforms where jobs are executed in a non-shared file system environment.

\section{Experimental Settings}

Most SWfMSs assume that activities must be executed isolated, one per available CPU or core. The multitenant architecture of cloud computing platforms does not provide the same level of isolation expected by HPC applications: the same physical machine can host more that one virtual machine without the user knowledge. We argue that on those platforms a moderate use of shared multiprocessors can actually improve the performance and decrease the cost of the execution. In the remaining of the text we will evaluate the execution of two real-world scientific applications in a public cloud computing platform.

\subsection{Resource Management}

Within Pegasus, HTCondor manages the available resources through slots of execution, in which tasks can be scheduled to perform their computation. Typically, HTCondor creates one slot per CPU-core. For example, in a platform composed of eight singlecore execution nodes, the resource manage will advertise eight slots of execution when using the default configuration. For this experiment, we configured HTCondor to create multiple slots from a single core. As a result, tasks can be mapped into slots within the same core, i.e. task executions will share the same CPU core. Memory and disk partitions can also be configured among the slots, however the analysis of the performance impact of disk and memory requirements is out of the scope of this work. Thus, we rely in the default configuration, which distributes them equally. 


\subsection{Scientific Applications}

Montage (Figure 2a): A workflow created by the NASA Infrared Processing and Analysis Center as an open-source toolkit to generate custom mosaics of astronomical images [Jacob et al. 2009]. In the workflow, the geometry of the output mosaic is calculated from the input images. The inputs are then re-projected to have the same spatial scale and rotation, the level of background emissions in all images is uniformed, and the reprojected, corrected images are co-added to form the output mosaic. The size of the workflow depends on the number of input images of the mosaic.

Epigenomics (Figure 2b): A workflow that automates the execution of genome sequencing operations developed at the USC Epigenome Center [epi 2015] to map the epigenetic state of human cells on a genome-wide scale. The workflow processes multiple sets of genome sequences in parallel. These sequences are split into subsets (chunks), that are posteriorly filtered to remove noisy and contaminating sequences, reformatted, and then mapped to a reference genome. The mapped sequences are finally merged and indexed for later analysis. The size of the workflow depends on the chunking factor used on the input data.

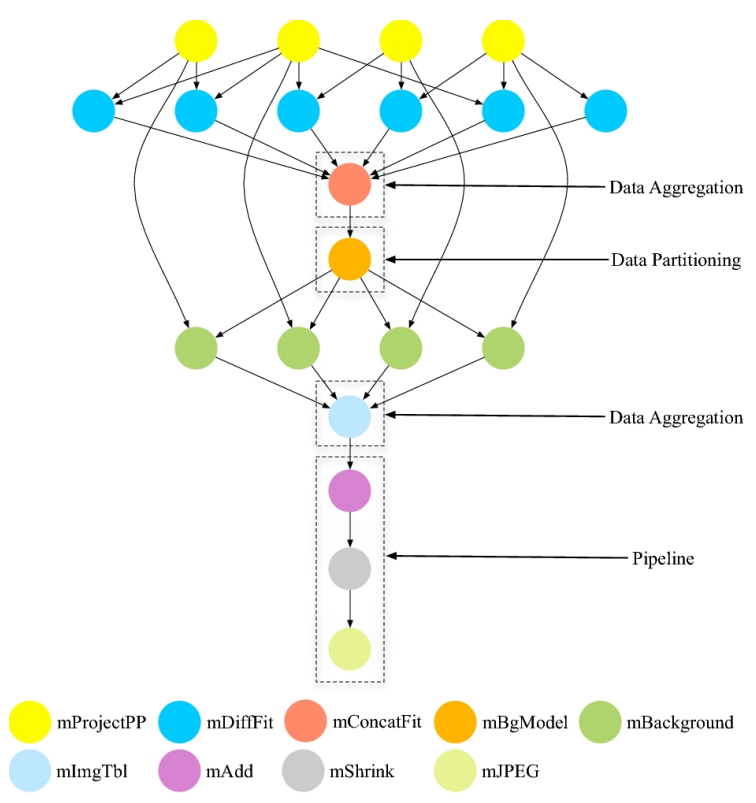

(a) Montage

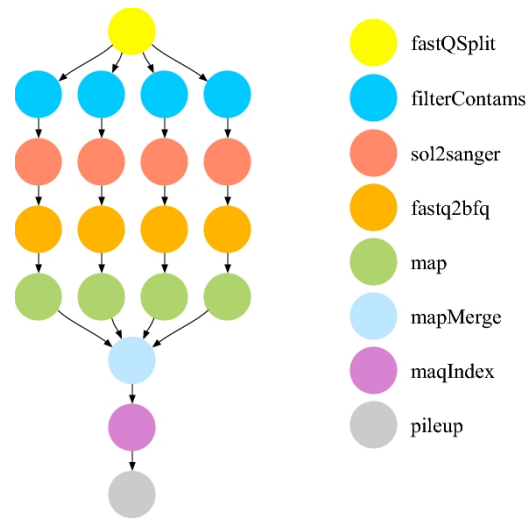

(b) Epigenomics

Figure 2. Examples of instances of the workflows used in the experiments.

\subsection{Experiment Conditions}

Workflows were executed on the Google Compute Engine (GCE), the Infrastructure as a Service (IaaS) component of Google Cloud Platform [goo 2015]. For each workflow run, we deployed a HTCondor pool of VMs by using PRECIP [Azarnoosh et al. 2013], an experiment management tool that automates resource provisioning of cloud resources.

In this experiment, we used $n l$-standard- 1 instance type. In an $n l$ series, a virtual CPU is implemented as a single hardware hyper-thread on a $2.6 \mathrm{GHz}$ Intel Xeon E5 (Sandy Bridge), 2.5GHz Intel Xeon E5 v2 (Ivy Bridge), or 2.3GHz Intel Xeon E5 v3 (Haswell). 
An n1-standard-1 machine is composed of one virtual CPU of 2.75 GCEUs (Google Compute Engine Units) and 3.75GB of memory. The image used to instantiate the VMs operates over Ubuntu 15.04 and uses Pegasus 4.5.0 as the SWfMS, and HTCondor 8.3.5 as the resource manager. We deployed 9 instances of the $n 1$-standard-1 machine, where one was used as a central node (submit node), and the remaining instances as execution nodes.

Runs of each workflow instance were performed with different datasets or input parameter options. For the Montage workflow, a single dataset was used, and the degree input parameter was set to $0.1,0.5,1.0,2.0$, and 4.0 degrees, which leverages its size (in terms of number of tasks). Table 1 shows the number of tasks per type for the Montage workflow. Two different input datasets (TAQ and HEP) were used for the Epigenomics workflow, which the number of tasks per task type are shown in Table 2.

\begin{tabular}{lrrrrr} 
Task & $\mathbf{0 . 1}$ & $\mathbf{0 . 5}$ & \multicolumn{1}{c}{} & $\mathbf{2 . 0}$ & \multicolumn{4}{c}{$\mathbf{0}$} \\
\hline mAdd & 1 & 1 & 1 & 1 & 5 \\
mBackground & 8 & 32 & 84 & 300 & 603 \\
mBgModel & 1 & 1 & 1 & 1 & 1 \\
mConcatFit & 1 & 1 & 1 & 1 & 1 \\
mDiffFit & 13 & 73 & 213 & 836 & 2316 \\
mImgtbl & 1 & 1 & 1 & 1 & 5 \\
mJPEG & 1 & 1 & 1 & 1 & 1 \\
mProjectPP & 8 & 32 & 84 & 300 & 802 \\
mShrink & 1 & 1 & 1 & 1 & 4
\end{tabular}

Table 1. Number of tasks per task type for the Montage workflow for different values of the degree parameter.

\begin{tabular}{lrr} 
Task & TAQ & HEP \\
\hline chr21 & 1 & 1 \\
fast2bfq & 729 & 1360 \\
fastqSplit & 2 & 7 \\
filterContams & 729 & 1360 \\
map & 729 & 1360 \\
mapMerge & 3 & 8 \\
pileup & 1 & 1 \\
sol2sanger & 729 & 1360
\end{tabular}

Table 2. Number of tasks per task type for the Epigenomics workflow for the TAQ and HEP datasets.

In each execution of a workflow instance, each core of the execution nodes was partitioned into 1, 2, 4, or 8 slots. Therefore, each workflow run had 8 cores and between 8 to 64 total slots (i.e., $8 \times n$ slots, where $n$ denotes the number of partitions). Table 3 summarizes the experimental settings.

We characterize execution profiles for the scientific workflows described in the previous section by using the Kickstart [Vockler et al. 2006, Juve et al. 2015] profiling tool from Pegasus. Kickstart monitors and records information about the execution of individual workflow tasks. It captures fine-grained profiling data such as process I/O, runtime, memory usage, and CPU utilization. Workflow profiles are then used to support the analyses of the experiment results. 


\begin{tabular}{lccrc} 
Workflow & Case & Partitions & Total Input Size & Number of Executions \\
\hline Montage & 0.1 & $2,4,8$ & $12.41 \mathrm{MB}$ & 18 \\
Montage & 0.5 & $2,4,8$ & $49.79 \mathrm{MB}$ & 18 \\
Montage & 1.0 & $2,4,8$ & $130.43 \mathrm{MB}$ & 18 \\
Montage & 2.0 & $2,4,8$ & $489.75 \mathrm{MB}$ & 18 \\
Montage & 4.0 & $2,4,8$ & $1.81 \mathrm{~GB}$ & 18 \\
Epigenomics & TAQ & $2,4,8$ & $1.01 \mathrm{~GB}$ & 18 \\
Epigenomics & HEP & $2,4,8$ & $1.79 \mathrm{~GB}$ & 18
\end{tabular}

Table 3. Experimental settings. The number of partitions denote the number of slots advertised by the resources.

\section{Results and Discussion}

In this section, we evaluate the impact of CPU-core partitioning on the overall performance of the workflow executions. We also investigate the performance gain of individual workflow tasks. Experiment results for 1 partition (i.e., no resource sharing) are used as the baseline for comparison.

Figure 3 shows the average walltime for both workflows. The walltime represents the turnaround time to execute all workflow tasks, which includes the execution of all computing jobs, and the extra jobs added by Pegasus for data management (e.g., data staging, cleanup tasks, and directory management). For the Montage workflow, the performance gain/loss is negligible for small degree instances $(0.1,0.5$, and 1.0$)$ due to the very low number of tasks, and thus very low degree of parallelism (see Table 1 and Figure 2a), to the short duration of its tasks (see Figure 4), and the overhead inherent to the workflow management system, e.g. timespan to release next task [Chen and Deelman 2011]. For higher degrees (2.0 and 4.0), the performance of the workflow execution is improved up to $9 \%$ as shown in Table 4. Figure 4 displays the average task runtime per task type and degree for the Montage workflow. Although the use of partitioned slots slightly impacts the runtime of individual tasks, the parallelism gain overcomes this loss.
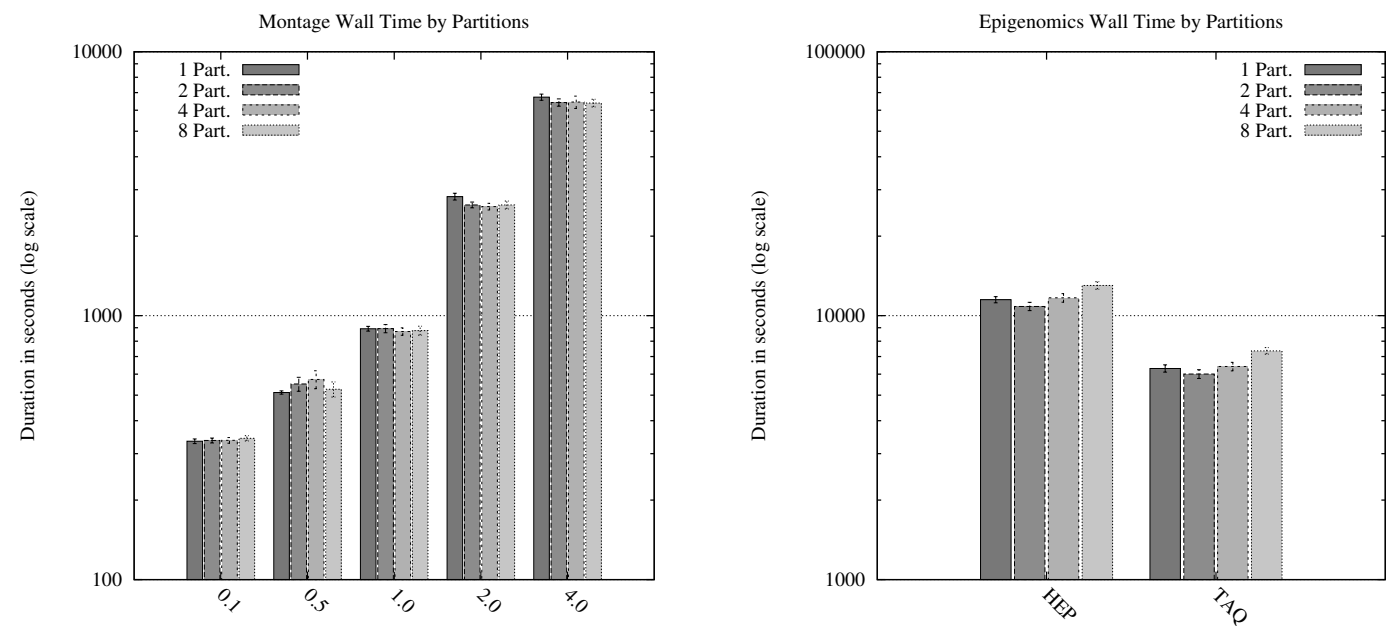

Figure 3. Workflow makespan evaluation for different partition sizes.

For the Epigenomics workflow, an overall performance gain up to $6 \%$ is observed for partitions of size 2. Epigenomics execution is mostly dominated by the 

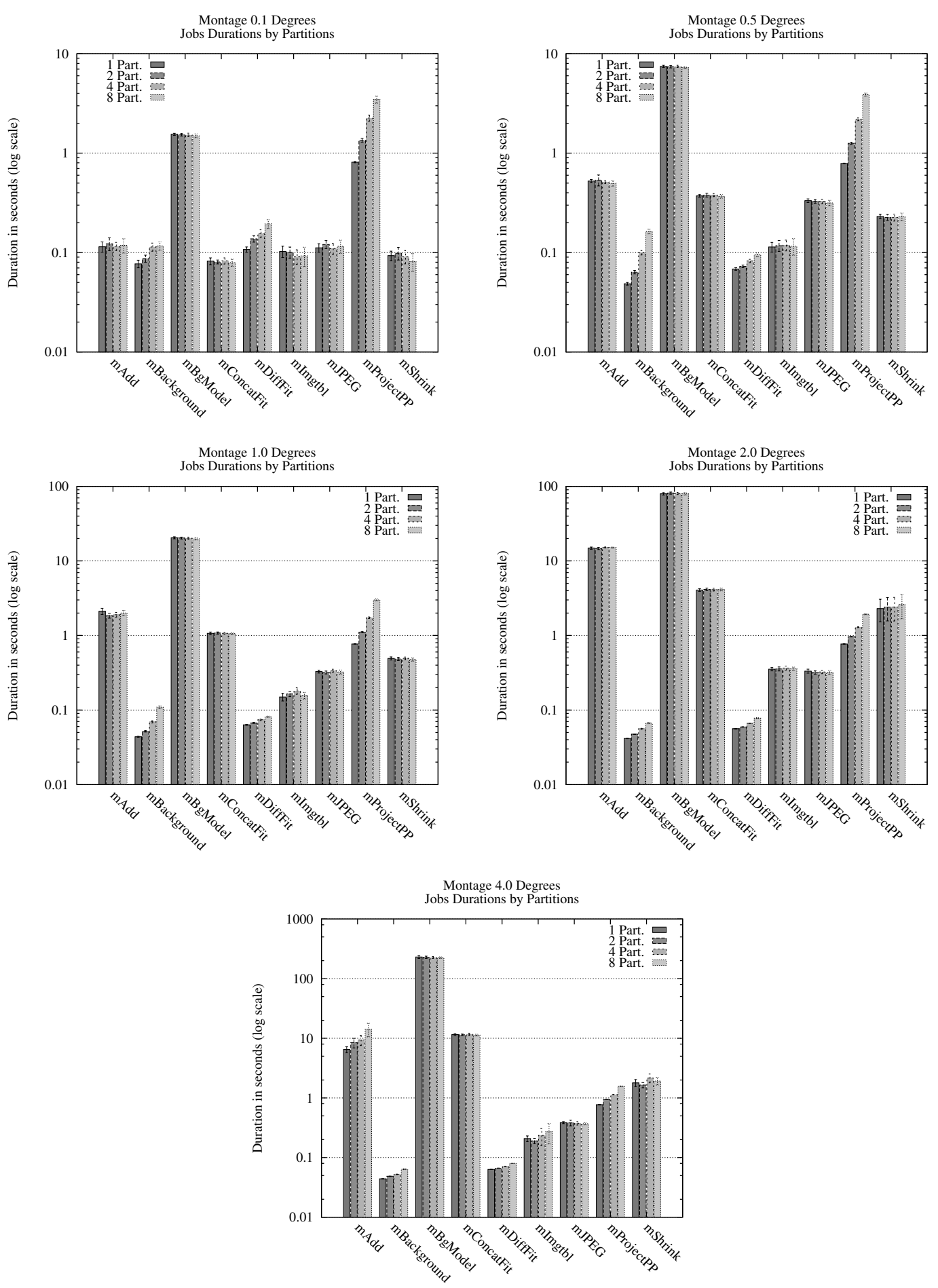

Figure 4. Average task runtime per task type and different degree values for the Montage workflow.

map task (Figure 5), which process a significant amount of data and is also CPUintensive [Juve et al. 2013, Ferreira da Silva et al. 2013]. A very large partitioning size significantly impacts the runtime of map tasks and consequently slows down the workflow execution. 

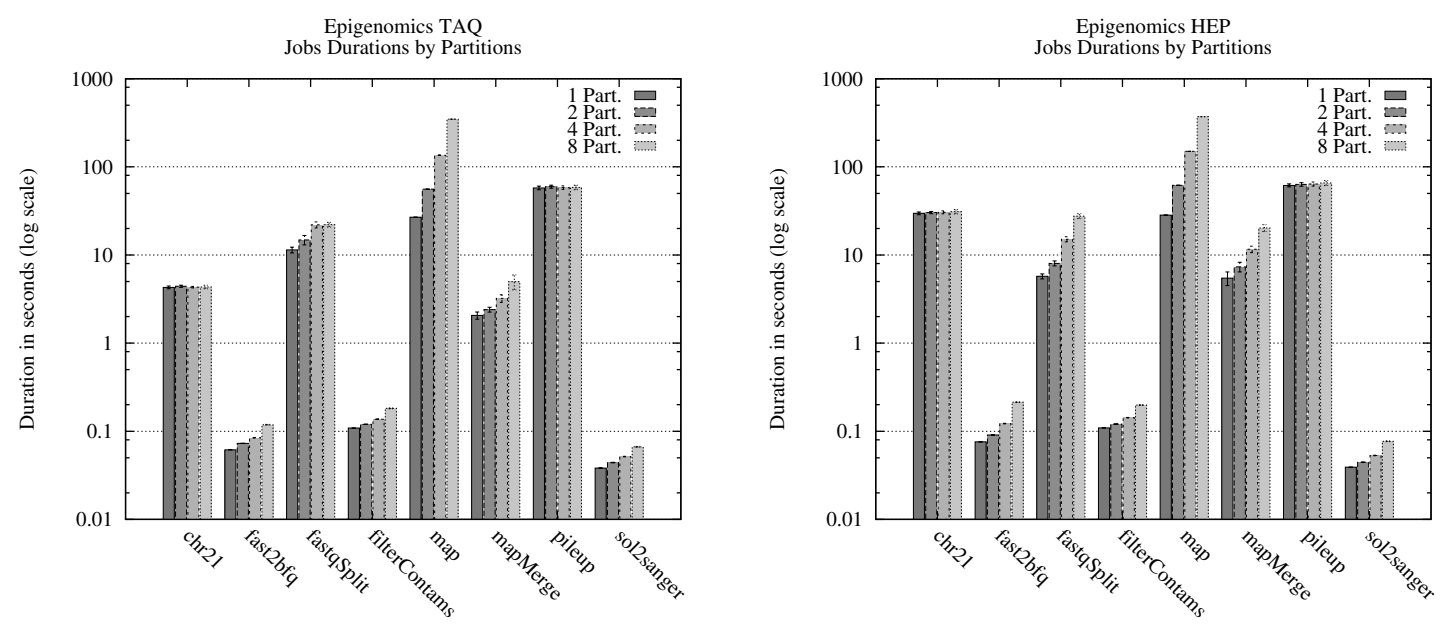

Figure 5. Average task runtime per task type and different degree values for the Epigenomics workflow.

The slowdown experienced by individual tasks is mainly due to the decrease of the CPU utilization. For each experiment case, we measured the average CPU usage rate $\bar{F}$ for each task type $T$, defined as follows:

$$
\bar{F}(T)=\frac{1}{|\mathcal{T}|} \times \sum_{t \in \mathcal{T}}\left(\frac{\text { CPU time }_{t}}{\text { runtime }_{t}}\right)
$$

where $\mathcal{T}$ is the set of all tasks of type $T$ within a workflow instance. The higher the value of $\bar{F}$ the higher is the CPU utilization (at most 1, which means 100\%). Figure 6 shows the average CPU utilization rate for the Montage (top) and Epigenomics (bottom) workflows. Note that for the Montage workflow only measurements for 2.0 and 4.0 degrees are shown since they yield noticeable performance gain. Overall, the Epigenomics workflow is CPU-intensive, while Montage workflow is predominantly I/Ointensive [Juve et al. 2013]. This result suggests that resource sharing optimizations for Epigenomics is limited to a small number of partitions as shown in Figure 5. Map tasks present an exponential decrease of CPU utilization as the number of partitions increase, which significantly affects the task runtime, and thereby slows down the workflow execution. On the other hand, linear CPU usage decreases (e.g., mBackground, mDiffFit, and $\mathrm{mPrOjectPP}$ for the Montage workflow), at the expense of slightly slowing down task executions, yield fair performance gains.

Table 4 summarizes the experimental results to evaluate the impact of shared multiprocessor (partitions) when running scientific workflows on cloud resources. The table highlights the average workflow makespan (walltime), and the average performance gains $(+)$ or losses (-) for different partition sizes.

\section{Concluding Remarks}

Cloud computing platforms are known by their performance unpredictability. Virtual machines allow cloud providers to make a more clever use of the physical resources, at the expense of hiding details about the physical machines from users. 

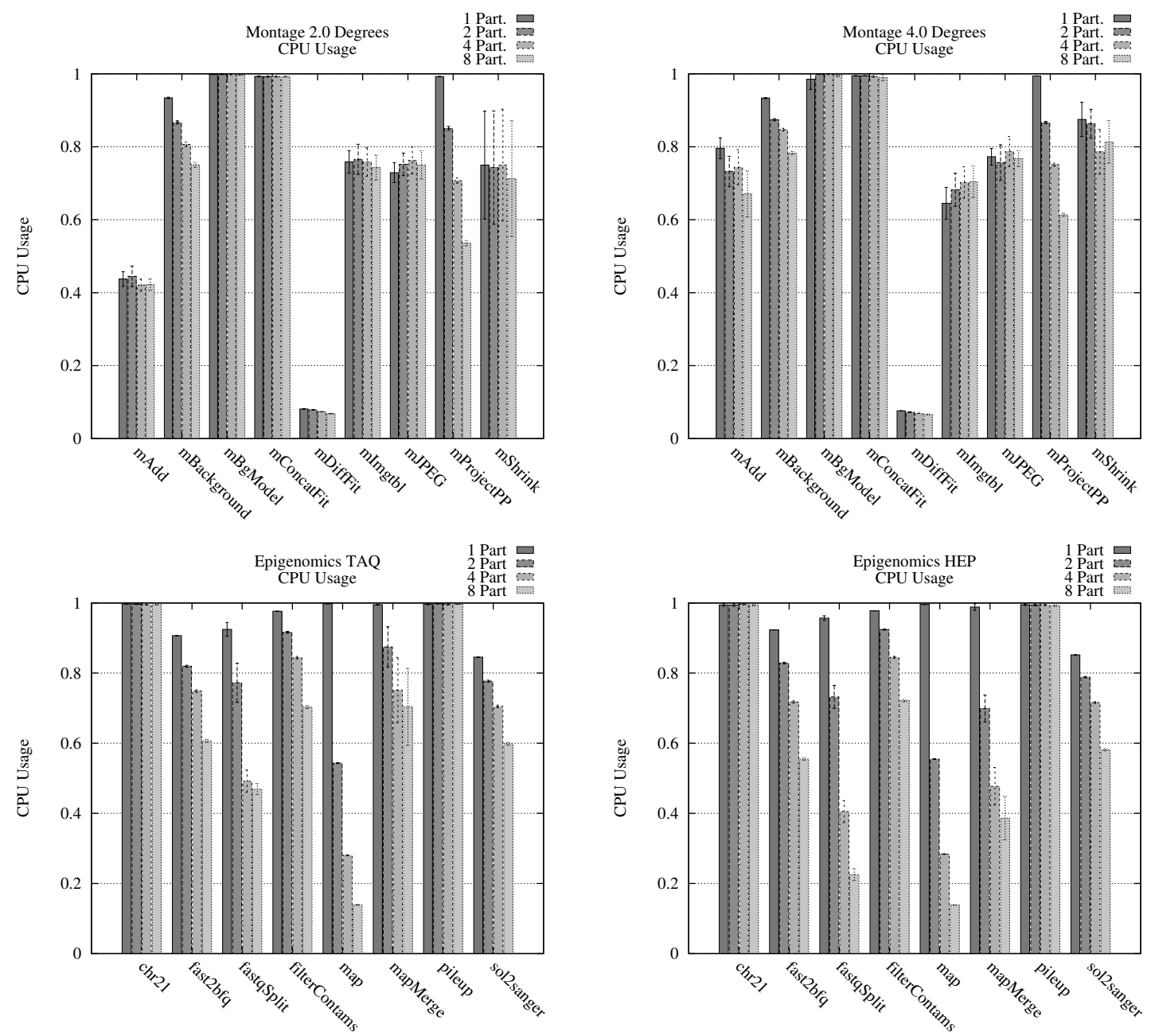

Figure 6. Average CPU utilization for the Montage (top) and Epigenomics (bottom) workflows per task type.

\begin{tabular}{lc|rrrrrrrr}
\multirow{2}{*}{ Workflow } & \multirow{2}{*}{ Case } & \multicolumn{2}{|c}{ Baseline } & \multicolumn{2}{c}{ 2 Partitions } & \multicolumn{2}{c}{ 4 Partitions } & \multicolumn{2}{c}{ 8 Partitions } \\
& & Walltime & Gain & Walltime & Gain & Walltime & Gain & Walltime & Gain \\
\hline Montage & 0.1 & 334.3 & $0 \%$ & 336.5 & $-1 \%$ & 336.9 & $-1 \%$ & 343.1 & $-3 \%$ \\
& 0.5 & 511.6 & $0 \%$ & 550.8 & $-7 \%$ & 573.4 & $-11 \%$ & 526.2 & $-3 \%$ \\
& 1.0 & 892.4 & $0 \%$ & 893.6 & $0 \%$ & 870.6 & $+3 \%$ & 877.5 & $+2 \%$ \\
& 2.0 & 2825.3 & $0 \%$ & 2627.9 & $+8 \%$ & 2589.4 & $+9 \%$ & 2627.8 & $+8 \%$ \\
\multirow{2}{*}{ Epigenomics } & 4.0 & 6730.0 & $0 \%$ & 6420.0 & $+5 \%$ & 6443.3 & $+4 \%$ & 6390.0 & $+5 \%$ \\
& TAQ & 6310.0 & $0 \%$ & 6010.0 & $+5 \%$ & 6416.0 & $-2 \%$ & 7356.7 & $-14 \%$ \\
& HEP & 11493.3 & $0 \%$ & 10840.0 & $+6 \%$ & 11683.3 & $-2 \%$ & 13020.0 & $-12 \%$
\end{tabular}

Table 4. Summary of performance gains for different partition sizes. The baseline is defined as runs with CPU-cores partitioned into a single slot, i.e. no resource sharing.

We have studied the indirect impact of this unpredictability on scientific applications, generally designed for HPC platforms. Using two real-world scientific workflows and a well-known public cloud provider, we have analyzed the consequences of executing multiple workflow tasks concurrently in a same core. 
In summary, shared multiprocessors within a single virtual machine can improve the execution of scientific workflows, however they should be sparingly used. Experimental results show that larger workflows benefit more from resource sharing. On public clouds, reducing the execution time also implies in lower costs due to lower lease times.

As expected, CPU intensive jobs are likely to suffer performance degradation. Thus, even if the workflow execution time can be improved, ideally the use of resource sharing must be considered in a case-by-case basis for each task.

As future work we are developing an automatic slot partitioning scheme. Using tasks profiling, a specialized scheduling can group low CPU tasks to take advantage of resource sharing. Also, dynamic allocation and configuration of resources can be mixed to attend specific needs of an ongoing workflow execution.

\section{Acknowledgments}

This research was funded by CNPq (process \#134403/2013-4), NAPSoL-PRP-USP and CAPES-Procad. We would like to thank Google for the awarded cloud platform credits that has made possible the experiments reported in this text. This research was also supported by the National Science Foundation under the $\mathrm{SI}^{2}-\mathrm{SSI}$ program, award number 1148515.

\section{References}

(2015). Google Cloud. https: / / cloud.google.com/. [Accessed 2015-07-31].

(2015). USC Epigenome Center. http: / / epigenome . usc . edu/. [Accessed 201507-31].

Azarnoosh, S., Rynge, M., Juve, G., et al. (2013). Introducing PRECIP: An API for managing repeatable experiments in the cloud. In The 2013 IEEE International Conference on Cloud Computing Technology and Science - Volume 02, CLOUDCOM '13, pages 19-26.

Callaghan, S., Maechling, P., Small, P., et al. (2011). Metrics for heterogeneous scientific workflows: A case study of an earthquake science application. Int. J. High Perform. Comput. Appl., 25(3):274-285.

Chen, W. and Deelman, E. (2011). Workflow overhead analysis and optimizations. In Proceedings of the 6th workshop on Workflows in support of large-scale science, pages 11-20. ACM.

Deelman, E., Juve, G., and Berriman, G. B. (2012). Using clouds for science, is it just kicking the can down the road? In The 2nd International Conference on Cloud Computing and Services Science, CLOSER '12, pages 127-134.

Deelman, E., Singh, G., Livny, M., Berriman, B., and Good, J. (2008). The cost of doing science on the cloud: The Montage example. In International Conference for High Performance Computing, Networking, Storage and Analysis, 2008, SC '08, pages 112.

Deelman, E., Vahi, K., Juve, G., et al. (2015). Pegasus, a workflow management system for science automation. Future Generation Computer Systems, 46(0):17-35. 
Fard, H. M., Prodan, R., Barrionuevo, J. J. D., and Fahringer, T. (2012). A multiobjective approach for workflow scheduling in heterogeneous environments. In The 12th IEEE/ACM International Symposium on Cluster, Cloud and Grid Computing, CCGRID '12, pages 300-309.

Ferreira da Silva, R., Juve, G., Deelman, E., et al. (2013). Toward fine-grained online task characteristics estimation in scientific workflows. In 8th Workshop on Workflows in Support of Large-Scale Science, WORKS '13, pages 58-67.

Frey, J. (2002). Condor dagman: Handling inter-job dependencies. University of Wisconsin, Dept. of Computer Science, Tech. Rep.

Giardine, B., Riemer, C., Hardison, R. C., et al. (2005). Galaxy: a platform for interactive large-scale genome analysis. Genome Res., 15(10):1451-1455.

Hoffa, C., Mehta, G., Freeman, T., et al. (2008). On the use of cloud computing for scientific workflows. In The IEEE Fourth International Conference on eScience, eScience '08, pages 640-645.

Jacob, J. C., Katz, D. S., Berriman, G. B., et al. (2009). Montage: a grid portal and software toolkit for science-grade astronomical image mosaicking. Int. J. Comput. Sci. Eng., 4(2):73-87.

Juve, G., Chervenak, A., Deelman, E., et al. (2013). Characterizing and profiling scientific workflows. Future Generation Computer Systems, 29(3):682-692.

Juve, G., Deelman, E., Vahi, K., et al. (2009). Scientific workflow applications on Amazon EC2. In The 5th IEEE International Conference on e-Science, e-Science '09, pages $59-66$.

Juve, G., Tovar, B., Ferreira da Silva, R., et al. (2015). Practical resource monitoring for robust high throughput computing. In Workshop on Monitoring and Analysis for High Performance Computing Systems Plus Applications, HPCMASPA'15, page to appear.

Ludäscher, B., Altintas, I., Berkley, C., et al. (2006). Scientific workflow management and the Kepler system. Concurr. Comput. : Pract. Exper., 18(10):1039-1065.

Malawski, M., Juve, G., Deelman, E., and Nabrzyski, J. (2012). Cost- and deadlineconstrained provisioning for scientific workflow ensembles in IaaS clouds. In The International Conference on High Performance Computing, Networking, Storage and Analysis, SC '12, pages 22:1-22:11.

Taylor, I., Deelman, E., Gannon, D., and Shields, M. (2007). Workflows for e-Science. Springer.

Thain, D., Tannenbaum, T., and Livny, M. (2005). Distributed computing in practice: The Condor experience. Concurr. Comput. : Pract. Exper., 17(2-4):323-356.

Vockler, J. S., Mehta, G., Zhao, Y., Deelman, E., and Wilde, M. (2006). Kickstarting remote applications. In International Workshop on Grid Computing Environments, GCE '06.

Wolstencroft, K., Haines, R., Fellows, D., et al. (2013). The Taverna workflow suite: designing and executing workflows of web services on the desktop, web or in the cloud. Nucleic Acids Research, 41(W1):W557-W561. 